INPLASY

PROTOCOL

To cite: Zhang et al. Efficacy of prehabilitation for colorectal cancer patients after surgery: a protocol for systematic review and meta-analysis. Inplasy protocol 202190007. doi: 10.37766/inplasy2021.9.0007

Received: 02 September 2021

Published: 02 September 2021

Corresponding author: Wenjun Zhang

gansumingyi@163.com

Author Affiliation:

Gansu Hospital of Traditional Chinese Medicine.

Support: Yes.

Review Stage at time of this submission: Preliminary searches.

Conflicts of interest: None declared.

\section{Efficacy of prehabilitation for colorectal cancer patients after surgery: a protocol for systematic review and meta-analysis}

\author{
Zhang, WJ1; Yang, M2; Yuan, Y3.
}

Review question / Objective: Our study aims to evaluate the effects of prehabilitation in colorectal cancer patients after surgery, meanwhile helps colorectal cancer patients to achieve a better condition.

Condition being studied: Colorectal cancer.

Information sources: The following databases, such as PubMed, Embase.com, the Cochrane Central Register of Controlled Trials (CENTRAL), Web of Science, China National Knowledge Infrastructure (CNKI), Wanfang, and SinoMed, will be used to retrieve literature. The search time is up to August 1, 2021. There are no limits on languages. A systematic retrieve strategy will be formulated to apply in the mentioned databases. Additionally, we will manually search journal articles, conference articles, and attached references as a supplement.

INPLASY registration number: This protocol was registered with the International Platform of Registered Systematic Review and Meta-Analysis Protocols (INPLASY) on 02 September 2021 and was last updated on 02 September 2021 (registration number INPLASY202190007).

\section{INTRODUCTION}

Review question / Objective: Our study aims to evaluate the effects of prehabilitation in colorectal cancer patients after surgery, meanwhile helps colorectal cancer patients to achieve a better condition.

Condition being studied: Colorectal cancer.

\section{METHODS}

Search strategy: The following databases, such as PubMed, Embase.com, the Cochrane Central Register of Controlled Trials (CENTRAL), Web of Science, China National Knowledge Infrastructure (CNKI), Wanfang, and SinoMed, will be used to retrieve literature. The search time is up to August 1, 2021. There are no limits on languages. A systematic retrieve strategy 
will be formulated to apply in the mentioned databases. Additionally, we will manually search journal articles, conference articles, and attached references as a supplement.

Participant or population: We will include individuals diagnosed with colorectal cancer (by pathological biopsy), meanwhile received colorectal surgery. The age limit is 18 to 80, with no restriction on gender, ethnicity, economic status, and education.

Intervention: There is no specific standardization about prehabilitation, but it mainly contains three sections: exercise, nutrition, and psychology. It is worth emphasizing that this intervention should execute before surgery, for any length of time. Exercise interventions include multitudinous modalities, regardless of environment, frequency, intensity, and materials.

Comparator: The comparison intervention is defined as routine preoperative preparation, usual care, standard care, and other conventional treatments.

Study designs to be included: Only researches classified into randomized controlled trials (RCTs) will be included. In terms of languages, publication time, and sample size, there are no restrictions.

Eligibility criteria: 2.2.1.Inclusion2.2.1.1. PopulationWe will include individuals diagnosed with colorectal cancer (by pathological biopsy), meanwhile received colorectal surgery. The age limit is $\mathbf{1 8}$ to $\mathbf{8 0}$, with no restriction on gender, ethnicity, economic status, and education. Emergency surgery, and preoperative cognitive disorder or multiple organ failure (MOF) will be excluded.2.2.1.2. InterventionThere is no specific standardization about prehabilitation, but it mainly contains three sections: exercise, nutrition, and psychology[5]. It is worth emphasizing that this intervention should execute before surgery, for any length of time. Exercise interventions include multitudinous modalities, regardless of environment, frequency, intensity, and materials.2.2.1.3. ComparisonThe comparison intervention is defined as routine preoperative preparation, usual care, standard care, and other conventional treatments. 2.2.1.4. OutcomesPrimary outcomes include the following: the time of first walking after surgery, recovery of gastrointestinal function after surgery, hospital length of stay, functional capacity, postoperative complications, mortality rates, and so on. 2.2.1.5. Type of studiesOnly researches classified into randomized controlled trials (RCTs) will be included. In terms of languages, publication time, and sample size, there are no restrictions.

Information sources: The following databases, such as PubMed, Embase.com, the Cochrane Central Register of Controlled Trials (CENTRAL), Web of Science, China National Knowledge Infrastructure (CNKI), Wanfang, and SinoMed, will be used to retrieve literature. The search time is up to August 1, 2021. There are no limits on languages. A systematic retrieve strategy will be formulated to apply in the mentioned databases. Additionally, we will manually search journal articles, conference articles, and attached references as a supplement.

Main outcome(s): Primary outcomes include the following: the time of first walking after surgery, recovery of gastrointestinal function after surgery, hospital length of stay, functional capacity, postoperative complications, mortality rates, and so on.

Quality assessment / Risk of bias analysis: We will use the "Cochrane bias risk assessment tool"[19] to assess the risk of bias in the include studies. The risk of bias will be evaluated mainly according to the following domains: generation of random sequence (selection bias), allocation concealment (selection bias), blinding of participants and researchers (performance bias), blinding of outcome assessment (detection bias), integrity outcome data (attrition bias), selective reporting (reporting bias), other sources of bias (other bias). Two reviewers will 
independently assess the risk of bias. In case of dissensions, another reviewer will engage in to reach an agreement. Each item will be identified as low risk, high risk, and unclear risk.

Strategy of data synthesis: Automatic tool Stata (13.0; Stata Corporation, College Station, TX) will be used to implement data analysis. Meta-analyses will be conducted with the inverse variance method. For dichotomous variables, we will calculate the relative risks (RRs) and their 95\% confidence interval (Cl); for the continuous variable, we will calculate the mean difference (MD) and $95 \% \mathrm{Cl}$. The statistically significant level is set at $P<$ 0.05 . If it was not possible to conduct a meta-analysis, we will describe the synthesis methods or summary approach used.

Subgroup analysis: If the necessary data are available, subgroup analysis and metaregression will be done on different characteristics (age, gender, staging of patients, frequency of exercise, research design, evaluation of blindness) to explore the causes of heterogeneity.

Sensitivity analysis: Sensitivity analysis can be used to observe which of these factors (characteristics of studies or statistical method) will contribute to the synthesis results, meanwhile judge the robustness of our results.

\section{Language: English.}

\section{Country(ies) involved: China.}

Keywords: Prehabilitation, Colorectal cancer, Effectiveness, Systematic review, Meta-analysis.

Contributions of each author:

Author 1 - Wenjun Zhang.

Author 2 - Mei Yang.

Author 3 - Yuan Yuan. 